\title{
Optimal placement of grid-connected photovoltaic generators in a power system for voltage stability enhancement
}

\author{
Zetty Adibah Kamaruzzaman, Azah Mohamed, Ramizi Mohamed \\ Centre for Integrated Systems Engineering and Advanced Technologies (Integra), \\ Faculty of Engineering and Built Environment, Universiti Kebangsaan Malaysia, Bangi, 43600, Malaysia
}

\begin{tabular}{l}
\hline \hline Article Info \\
\hline Article history: \\
Received May 28, 2018 \\
Revised Oct 10, 2018 \\
Accepted Oct 25, 2018 \\
\hline
\end{tabular}

Keywords:

Optimal location

Photovoltaic generation

Wind driven optimization

\begin{abstract}
The high penetration of photovoltaic (PV) generation can cause many technical issues such as power quality and impact on the power system voltage stability. To improve voltage stability and reducing power loss in a power system with PV generators, appropriate planning of PV generators is considered by optimal placement of PV. Thus, an effective heuristic optimization technique such as the Wind Driven Optimization (WDO) technique is applied for determining optimal location of PV generators in a power system. For determining the optimal location of PV, the objective function considers maximizing the Improved Voltage Stability Index. The proposed method for optimal location of PV generators is implemented on the IEEE 118 and 30 bus transmission systems and the 69-radial distribution system. The optimization results show that integrating PV into the test systems improves voltage stability in the system. Comparing the performance of the WDO with the particle swarm optimization technique, it is shown that the WDO technique gives faster convergence.
\end{abstract}

Copyright () 2019 Institute of Advanced Engineering and Science. All rights reserved.

\section{Corresponding Author:}

Ramizi Mohamed,

Centre for Integrated Systems Engineering and Advanced Technologies (Integra),

Faculty of Engineering and Built Environment,

Universiti Kebangsaan Malaysia,

Bangi, 43600, Malaysia.

Email: ramizi@ukm.edu.my

\section{INTRODUCTION}

The increasing growth of load demand, lack of active power generation as well as technical constraints in power system are the challenges faced by power utilities in providing reliable power to consumers. Increase in load demands from the industrial, commercial and residential sectors has caused congestion in power network and this situation leads to voltage instability in power system [1]. Unstable power system due to voltage decline may lead to voltage collapse. Therefore, maintaining voltage stability is a major concern in power system operation. An approach to improve voltage stability in power system is by integrating renewable energy based distributed generations (DGs) which are usually installed in power distribution system [2-5]. However, the integration of renewable energy based DGs into a power system causes certain technical effects because the network design initially does not consider the integration of DG. The rapid growth of DG installations and high penetration of DG can cause many technical issues such as power quality. Integrating DG at random locations may cause increased power losses and voltage instability]. To maintain voltage stability and reduce power loss, it is important to place DGs optimally in a power system.

Several methods have been developed for determining optimal DG placement using either analytic or heuristic optimization approaches. In [6], power stability index is used to determine the critical buses in a system where these buses are considered as optimal DG locations. A modified voltage index is used as an 
objective function to improve voltage stability margin and mixed-integer nonlinear programming is applied to solve the optimization problem [7]. In addition, the voltage stability index (VSI) has been developed to determine the optimal placement and sizing of DG in a radial distribution system considering load growth [8]. Among the applied heuristic optimization techniques, the particle swarm optimization (PSO) has been used for determining the size and location of multi DGs considering multi-objective index [9]. In addition, considering heuristic optimization, the cuckoo search algorithm [10] and a constructive heuristic algorithm [11] have been used for solving the optimal DG placement problem. In all the above-mentioned methods, a general DG model with real power injection has been considered without considering any renewable energy.

One type of DG that is widely used in modern power system is the photovoltaic based distributed generation (PVDG). The PVDG system provides additional active power into the network, thereby facilitating reverse power flow and reducing energy consumption. The reactive power is solely provided by the conventional generators and therefore, reactive power limitation is neglected when finding the optimal PVDG location and sizing. To improve voltage stability margin and reducing power loss in a power system with PVDGs, appropriate planning of PVDGs is considered by optimal placement and sizing of PV. Some research works have been done for determining optimal placement and sizing of PVDG considering voltage stability improvement [12-14]. In [12], the PSO technique was used to determine optimum placement of PVDG and wind turbine by incorporating voltage stability factor. In [13], the artificial bee-colony optimization technique is developed for determining optimal sizing of hybrid PV/wind turbine/fuel cell system by maximizing the voltage collapse point using the VSI. In [14], the self-correction algorithm was used in determining optimal sizing of multiple PV and battery storage units considering voltage stability margin.

In this paper, a relatively new heuristic optimization technique known as wind driven optimization (WDO) [15] is applied for solving the optimal placement of PVDG units. In the proposed optimization problem formulation, the objective function is to maximize the voltage stability margin. Voltage stability of a power system is assessed by using a relatively new voltage stability index known as the improved voltage stability index (IVSI) [16]. Thus, for determining the optimal location of PVDG, the objective function considers maximizing the IVSI.

\section{THE MATERIAL AND METHOD}

This section describes IVSI for voltage stability analysis, PVDG modelling, and application of WDO for solving the optimization problem. The IVSI is an indicator for assessing whether a power system is stable from the voltage stability viewpoint. It is used in the objective function in finding the optimal location of PVDG for improving voltage stability in a power system. This section also discusses on the application of WDO technique in determining PVDG optimal placement. In this study, the entire modeling and simulation is carried out using MATLAB software.

\subsection{IVSI for voltage stability assessment}

The development of IVSI for voltage stability assessment is based on the current and power flow at a bus in a power network. The mathematical formula for IVSI for n-bus system is derived in [16] and it is given by,

$$
I V S I=\frac{-4 \sum_{j=0}^{n}\left(G_{i j}-B_{i j}\right)\left(P_{i}+Q_{i}\right)}{\left[\sum_{j=1}^{n} \mid V_{j}\left[\left[G_{i j}\left(\cos \delta_{i j}+\sin \delta_{i j}\right)-B_{i j}\left(\cos \delta_{i j}-\sin \delta_{i j}\right)\right]\right]^{2}\right.} \leq 1.0
$$

where $P_{i}+Q_{i}$ is the load active and reactive powers at the receiving end bus, $V_{j}$ is the voltage at the sending end bus, $\delta_{i j}$ is the phase angle difference between sending end and receiving end buses and $G_{i j}$ and $B_{i j}$ are the elements in the bus admittance matrix.

The IVSI is an indicator to assess voltage stability at the buses in a power system. The IVSI has a value between 0 and 1 . A bus is said to be stable if the IVSI value is less than 1 (IVSI $<1$ ). However, a bus is said to experience a voltage collapse if the IVSI value equals to 1 . When running a simulation to assess voltage instability using IVSI, the loading margin at a bus is increased gradually until the IVSI value reaches approximately 1 . 


\subsection{IVSI for voltage stability assessment}

In this study, the static model of PVDG is based on a single-diode equivalent circuit [12] where the active power is expressed as,

$$
P_{P V}=N_{P V} \cdot V_{O C} \cdot I_{S C}
$$

in which,

$$
\begin{aligned}
I_{S C} & =\left[I_{S C, S T C}+K_{i}\left[T_{C}-T_{r e f}\right]\right] \frac{G}{1000} \\
V_{O C} & =V_{O C, S T C}+K_{v}\left[T_{C}-T_{r e f}\right] \\
T_{C} & =T_{A}+\frac{N C O T-20}{800} G
\end{aligned}
$$

where $G$ is the solar irradiance set at constant $1000 \mathrm{~W} / \mathrm{m}^{2}, T_{C}$ is the solar operating temperature, $V_{O C}$ is the open circuit voltage, $I_{S C}$ is the short circuit current, $N_{P V}$ is $\mathrm{PV}$ module number, $T A$ is the ambient temperature which sat at constant $35^{\circ} \mathrm{C}, N O C T$ is the nominal operating cell temperature, $K_{i}$ is the short circuit current coefficient, $K_{v}$ is the open circuit voltage coefficient, $T_{r e f}$ is the reference operating temperature at $25^{\circ} \mathrm{C}$. All the electrical characteristics at standard test condition (STC) are taken from the PV datasheet provided by the manufacturer.

\subsection{Formulation of the Optimization Problem}

The optimization problem formulation considers maximizing voltage stability margin of a power system. The search for optimal solution is subjected to the following objective function and technical constraints. To determine the optimal PVDG location, the weakest bus in a test system has to be determined by calculating IVSI at each load bus. From the mathematical formula in (7), the IVSI value is between 0 and 1 , such that if a bus has an IVSI value of 1 , it means that the bus is the weakest bus from voltage instability viewpoint. Thus, the IVSI at each load bus as an objective function need to be maximized for determining the weakest bus. The mathematical formula of objective function for optimal PVDG location is given by,

$$
f_{1}=\max \left\{I V S I_{i}\right\}
$$

The technical constraints considered in the optimization problem are the PVDG, voltage and power flow constraints. The PVDG is limited by the available power sources at any given location. Thus, it is necessary to constraint the capacity between its maximum and minimum level of active power, which is given by,

$$
P_{D G, \min } \leq P_{D G} \leq P_{D G, \max }
$$

The system operation should be kept within $\pm 5 \%$ of voltage deviation and the voltage constraint is given by,

$$
0.95 p u \leq V_{i} \leq 1.05 p u
$$

The power flow equations to be satisfied are given by,

$$
\begin{aligned}
& \sum P_{i, P V D G}=P_{i}+P_{i, D} \\
& \sum Q_{i, P V D G}=Q_{i}+Q_{i, D}
\end{aligned}
$$

in which, 


$$
\begin{gathered}
P_{i}=V_{i} \sum_{j=1}^{N} Y_{i j} V_{j} \cos \left(\delta_{i}-\delta_{j}-\theta_{i j}\right) \\
Q_{i}=V_{i} \sum_{j=1}^{N} Y_{i j} V_{j} \sin \left(\delta_{i}-\delta_{j}-\theta_{i j}\right)
\end{gathered}
$$

where $N$ is the number of bus, $P_{i, P V D G}$ and $Q_{i, P V D G}$ are the $\mathrm{PV}$ generated real and reactive powers at bus $i$, respectively and $P_{i, D}$ and $Q_{i, D}$ are the real and reactive power demands at bus $i$, respectively.

\subsection{Application of WDO for Optimal Location of PVDG}

WDO is based on the Newton's second low of motion which is used to describe the motion of air parcels on the earth's atmosphere [15]. In the WDO topology, the position of small air parcels with random velocities in an $\mathrm{N}$-dimensional space is updated based on the physical equations that govern large-scale atmospheric motion. The movement of an air parcel in one dimension is contributed from the movement of air parcels in another dimension. The velocity of air parcels is derived from the concept of an air parcel moving with the wind in which the updating velocity is given by,

$$
u_{\text {new }}=\left((1-\alpha) u_{\text {old }}\right)+g\left(-x_{\text {old }}\right)+\left[\left|\frac{P_{\max }}{P_{\min }}-1\right| R T\left(x_{\max }-x_{\text {old }}\right)\right]+\left[\frac{-c u_{\text {old }}^{\text {old dim }}}{P_{\text {old }}}\right]
$$

where $u_{\text {new }}$ and $u_{\text {old }}$ are the updated velocity and current velocity, respectively, $x_{\text {max }}$ is the distance from the highest-pressure point, $P_{\max }$ is the maximum pressure, $P_{\text {old }}$ is the pressure at the current location, $T$ is the temperature, $g$ is the gravitational acceleration, and $R, \alpha$, and $c$ are constants.

The third term of equation (13) is a force towards the location of maximum pressure which is represented by the global best location of the optimization problem. Once the new velocity is calculated, the position of air parcel will be updated using the following equation.

$$
x_{\text {new }}=x_{\text {old }}+\left(u_{\text {new }} \times \Delta t\right)
$$

where $x_{\text {old }}$ and $x_{\text {new }}$ are the current position and new position of the air parcel, respectively and the time step $\Delta \mathrm{t}$ is assumed as 1 .

The WDO is applied for solving optimal location of PVDGs and it is described in terms of a flowchart as shown in Figure 1. From the flowchart, the algorithm starts with the initialization stage where all input parameters are defined to establish the problem boundaries. After setting the input, the first step of the optimization problem is to find the optimal PVDG location. In this algorithm, the PVDG is represented as an air parcel. During the simulation, the pressure of each air parcel (objective function) is evaluated at its current position. Then, the population is ranked based on its pressure and the velocity is evaluated using equation (13). The position of the air parcel for the next iteration is updated using (14). This procedure continues until it reaches the maximum number of iteration. Finally, the best location of the last iteration is recorded as the optimization result.

\subsection{Application of PSO for Optimal Location of PVDG}

The PSO is an evolutionary computation algorithm based on a social-psychological metaphor [17]. In PSO topology, the best individual position of particle, $\mathrm{i}$ is represented by,

$$
\text { Pbest }_{i}=\left(\text { Pbest }_{i 1}+\text { Pbest }_{i 2}+\text { Pbest }_{i 3}+\ldots+\text { Pbest }_{\text {in }}\right)
$$

whereas the best position of the entire swarm in $\mathrm{n}$-dimensional search space is represented by

$$
\text { Gbest }_{i}=\left(\text { Gbest }_{i 1}+\text { Gbest }_{i 2}+\text { Gbest }_{i 3}+\ldots+\text { Gbest }_{\text {in }}\right)
$$

An individual in a swarm approaches the optimum through it present position, present velocity, previous experiences of Pbest and Gbest and the experience of its neighbours. The particle of new position and velocity are determined using the following equations, 


$$
\begin{aligned}
& X_{i}^{(k+1)}=X_{i}^{k}+\Delta t+v_{i}^{k+1} \\
& V_{i}^{(k+1)}=\omega V_{i}^{(k)}+c_{1} r_{1}\left(\text { Pbest }_{i}^{(k)}-X_{i}^{(k)}\right)+c_{2} r_{2}\left(\text { Gbest }_{i}^{(k)}-X_{i}^{(k)}\right)
\end{aligned}
$$

Where $i=1,2,3, \ldots, n$ is the number of dimension of each particle, $c_{1}$ and $c_{2}$ are weight factors, $r_{1}$ and $r_{2}$ are random number between $(0,1)$ and $k$ is the number of iteration times. The inertia weighting factor is represented by $\omega$ of the following equation,

$$
\omega=\omega \max -\frac{\omega \max -\omega \min }{\text { iter }_{k, \max }} \cdot \text { iter }_{k}
$$

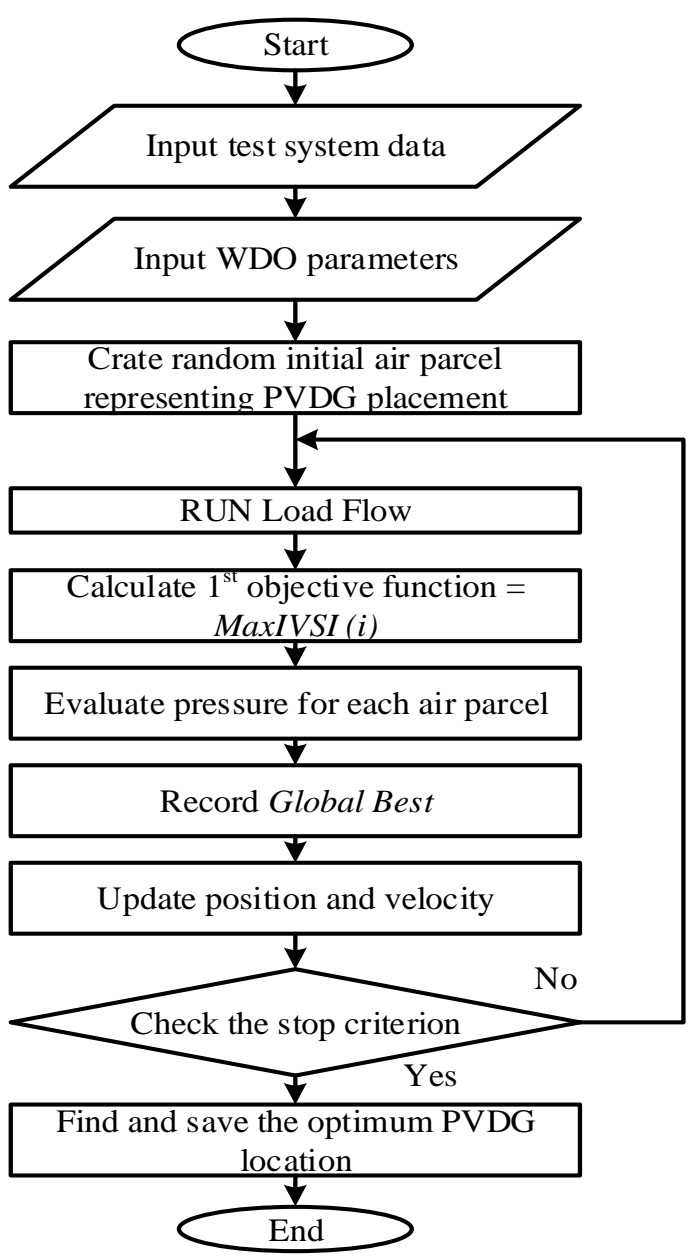

Figure 1. Implementation of WDO algorithm for solving optimal location of PVDG

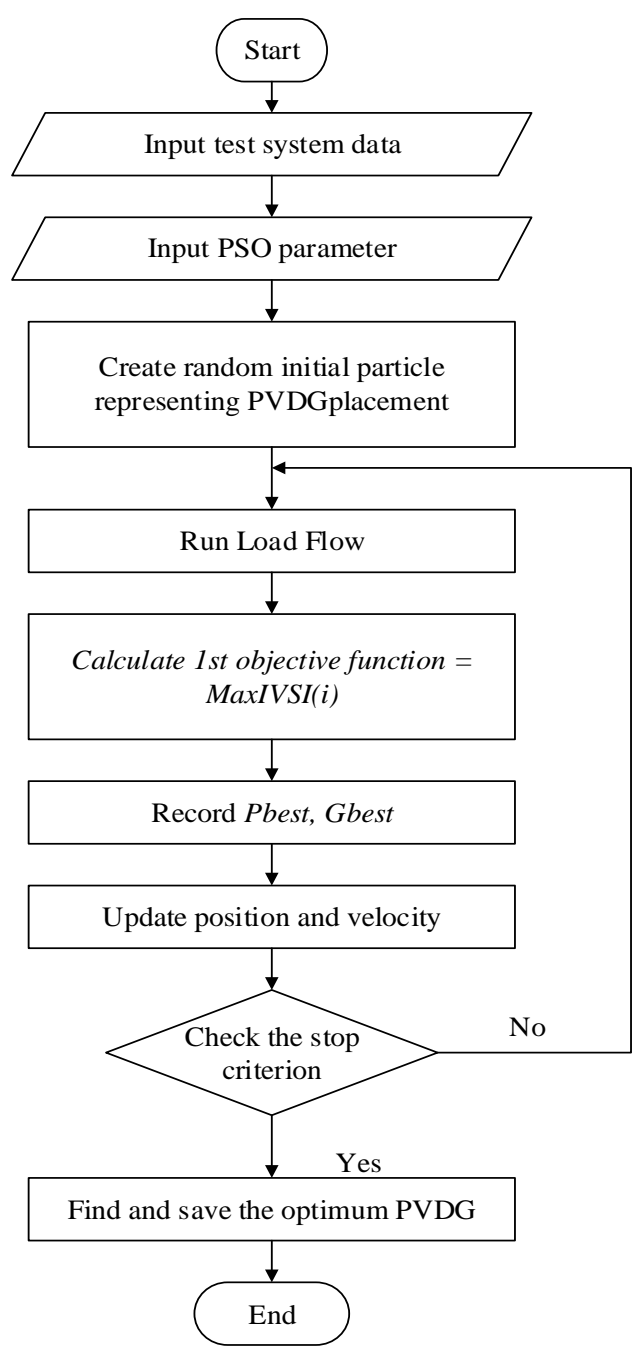

Figure 2. Implementation of PSO algorithm for solving optimal location of PVDG

The PSO technique is applied for solving optimal location of PVDGs and it is described in terms of a flowchart as shown in Figure 2. The first step of the optimization problem is to find the optimal PVDG location. In the PSO algorithm, the PVDG is represented as a particle in the swarm population. The algorithm starts by initializing all the input parameters as shown in the flowchart. During the simulation, $P_{\text {best }}$ (objective function) is evaluated at its current position using equation (15) whereas $G_{b e s t}$ is evaluated using equation (16). The new position and velocity of particle are updated using equation (17) and (18). This procedure continues until it reaches the maximum number of iteration. Finally, $G_{b e s t}$ of the last iteration is recorded as the optimization result. 


\section{RESULTS AND DISCUSSION}

The simulation results for optimal placement of PVDGs in the IEEE 30 bus, IEEE 118 bus and 69radial distribution systems using WDO and PSO are presented and discussed. Simulations were carried out to determine the weak buses in the test systems using IVSI. During the simulation, each load bus is evaluated individually by maximizing the IVSI as the objective function. The load factor is increased by a certain percentage of the base value until IVSI approaches 1, which is the voltage collapse point. The first bus that experiences voltage collapse is said to be the weakest bus in the system and it is marked as an optimal PVDG location. Thus, the PVDG is installed at this bus. For multiple numbers of PVDG, the first PVDG will be integrated using the bus ranking approach. The first PVDG will be integrated at the weakest bus (e.g. Bus 61 for the 69-radial distribution system) whereas the second PVDG will be integrated at the second weakest bus (e.g. Bus 64 for the 69-radial distribution system). Similar procedure is applied for the IEEE 30 bus system and IEEE 118 bus system.

Table 1, Table 2 and Table 3 show the IVSI values with variable load factors for the 69-radial distribution system, IEEE 30 bus, and IEEE 118 bus, respectively. The load factor of each bus is increased in percentage from the base load value. Results in Table 1 show that the optimal PVDG location is Bus 61 because at load factor of $190 \%$, the IVSI value equals 1.0 and Bus 61 is said to experience a voltage collapse. However, other buses in the system still operate within voltage stability limit with IVSI values less than 1.

Results in Table 2 show that the optimal PVDG location of the IEEE 30 bus system is Bus 30. It is the first bus to reach voltage collapse point (IVSI=1) at a load factor of $150 \%$. The next optimal PVDG location is Bus 26 and then followed by Bus 5.

Table 1. IVSI with load increment for the 69-radial distribution system

\begin{tabular}{ccccccc}
\hline \multirow{2}{*}{ Load Factor $(\%)$} & Bus 65 & Bus 64 & Bus 61 & Bus 65 & Bus 64 & Bus 61 \\
\hline 100 & 0.2622 & 0.4092 & 0.4316 & 0.2622 & 0.4092 & 0.4316 \\
120 & 0.3286 & 0.5127 & 0.5400 & 0.3286 & 0.5127 & 0.5400 \\
140 & 0.4079 & 0.633 & 0.6635 & 0.4018 & 0.6266 & 0.6591 \\
160 & 0.5211 & 0.7714 & 0.8003 & 0.4833 & 0.7534 & 0.7910 \\
180 & 0.5936 & 0.9100 & 0.9718 & 0.5750 & 0.8960 & 0.9389 \\
190 & 0.7093 & 0.9842 & 1.0 & 0.6256 & 0.9746 & 1.0 \\
\hline
\end{tabular}

Table 2. IVSI with load increment for the IEEE 30 bus system

\begin{tabular}{ccccccc}
\hline \multirow{2}{*}{ Load Factor $(\%)$} & \multicolumn{3}{c}{ WDO } & \multicolumn{3}{c}{ PSO } \\
& Bus 5 & Bus 26 & Bus 30 & Bus 5 & Bus 26 & Bus 30 \\
\hline 100 & 0.3682 & 0.1600 & 0.2057 & 0.3500 & 0.1479 & 0.1959 \\
120 & 0.1801 & 0.3057 & 0.3191 & 0.4417 & 0.2371 & 0.3140 \\
140 & 0.5984 & 0.4473 & 0.5894 & 0.5589 & 0.4237 & 0.5772 \\
150 & 0.6703 & 0.7019 & 1.0 & 0.6583 & 0.6958 & 1.0 \\
\hline
\end{tabular}

Results of the IEEE 118 bus system depicted Table 3 show that the first bus to experience voltage collapse is Bus 117 and then followed by Bus 1 and Bus 13. Thus, Bus 117 is considered as the optimal location for PVDG. From the results, each test system gives similar optimal PVDG location using the two different optimization techniques; WDO and PSO. However, the IVSI value at each load factor varies with different optimization techniques. Evaluation for the best optimization technique will not be performed considering that the differences in the IVSI values are not significant. However, the two different optimization techniques; WDO and PSO are compared in terms of convergence characteristic. Figures 3, 4 and 5 show the convergence characteristic for optimum PVDG location for the 69 radial-distribution system, 30 bus system and 118 bus system, respectively. The results in the figures show that the WDO gives faster convergence with minimum objective function values compared to the PSO technique.

Table 3. IVSI with load increment for the IEEE 118 bus system

\begin{tabular}{ccccccc}
\hline \multirow{2}{*}{ Load Factor (\%) } & WDO & & & PSO & \\
& Bus 13 & Bus 1 & Bus 117 & Bus 13 & Bus 1 & Bus 117 \\
\hline 100 & 0.0324 & 0.0102 & 0.0895 & 0.0324 & 0.0102 & 0.0895 \\
120 & 0.1106 & 0.0905 & 0.1407 & 0.1010 & 0.0837 & 0.1347 \\
140 & 0.1265 & 0.1177 & 0.1632 & 0.1196 & 0.1014 & 0.1531 \\
160 & 0.3143 & 0.2904 & 0.3879 & 0.3021 & 0.282 & 0.3733 \\
165 & 0.8379 & 0.9115 & 1.0 & 0.8261 & 0.8959 & 1.0 \\
\hline
\end{tabular}




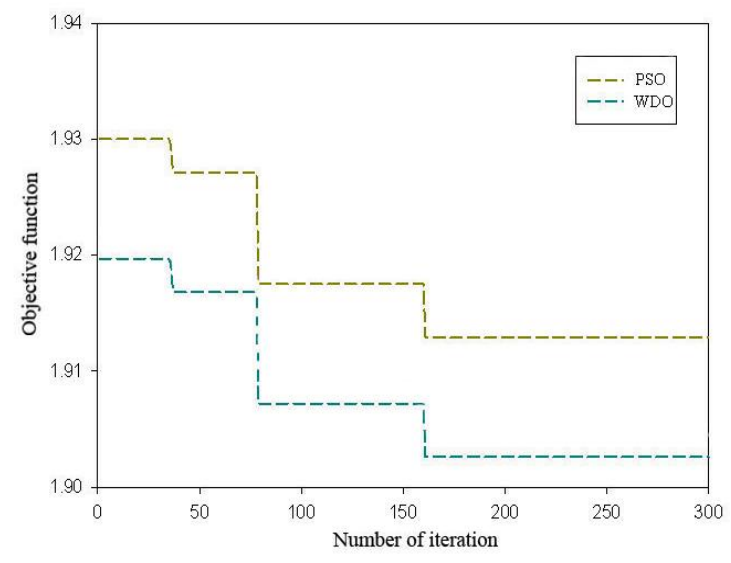

Figure 3. Convergence characteristic for optimum PVDG location using WDO and PSO for the 69 bus radial distribution system

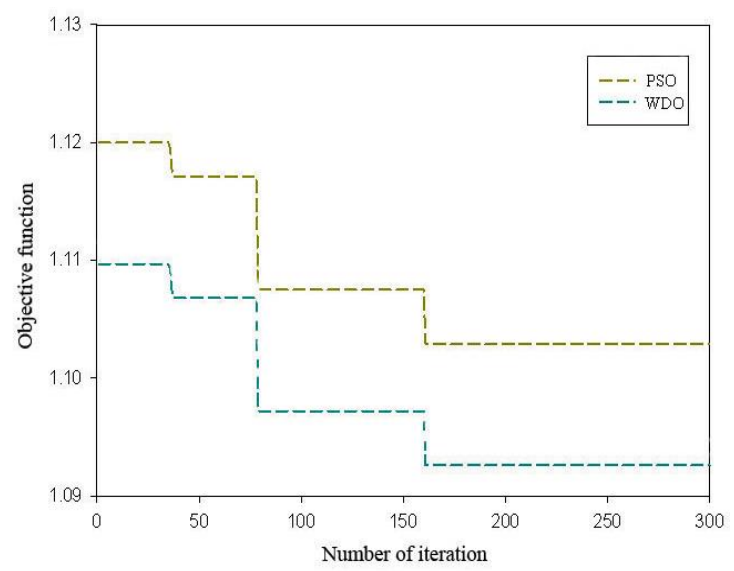

Figure 4. Convergence characteristic for optimum PVDG location Using WDO and PSO for the IEEE 30 bus system

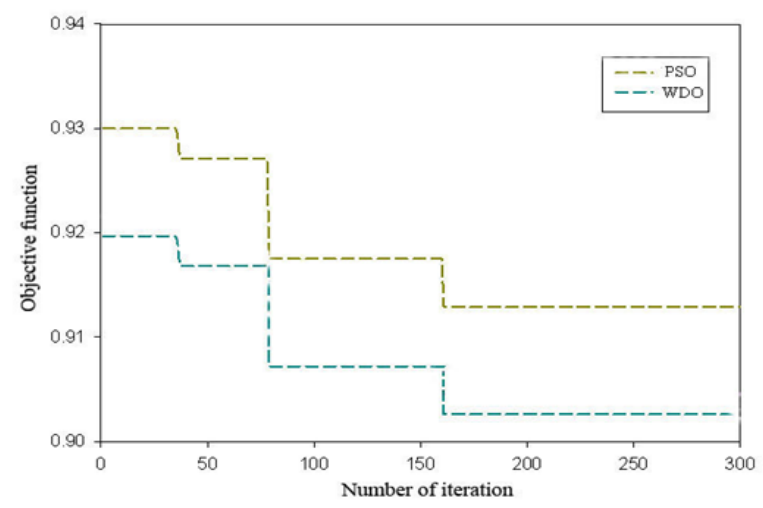

Figure 5. Convergence characteristic for optimum PVDG location using WDO and PSO for the IEEE 118 bus system

Table 4 shows the MATLAB time simulation for the implementation of WDO and PSO techniques in determining optimal location of FVTG generator. The WDO technique has faster time simulation compared to the PSO technique where differences can be seen for the IEEE 30 bus and 69 bus system simulations. Therefore, it can be concluded that the WDO technique is most suitable to be used in PVDG optimal location determination.

Table 4. MATLAB simulation time for optimal location of PVDG

\begin{tabular}{cccc}
\hline \multirow{2}{*}{ Optimization Technique } & \multicolumn{3}{c}{ MATLAB simulation time (s) } \\
& IEEE 118 bus & IEEE 30 bus & 69 bus system \\
\hline WDO & 816.703 & 805.418 & 804.531 \\
PSO & 816.831 & 810.924 & 807.999 \\
\hline
\end{tabular}

\section{CONCLUSIONS}

This paper has presented the application of WDO for determining optimal PVDG location by maximizing voltage stability margin, IVSI as an objective function. The WDO is applied to solve the optimization problem and it is compared with the PSO technique. The WDO and PSO techniques are tested on the IEEE 30 bus system, IEEE 118 bus system and 69-radial distribution system. Comparison on the performance of the WDO and PSO techniques showed that the WDO technique gives faster convergence compared to the PSO technique. Analysis of results proved that integration of PVDG on the weakest bus could improve power system voltage stability by increasing the value of IVSI. Increased PVDG penetration

\footnotetext{
Optimal placement of grid-connected photovoltaic generators in a power ... (Zetty Adibah Kamaruzzaman)
} 
level also increases voltage stability and improves bus voltage profiles. The maximum level of PVDG penetration is determined by ensuring that the bus voltage does not exceed the upper voltage limit of the nominal voltage.

\section{ACKNOWLEDGEMENT}

We would like to thank Universiti Kebangsaan Malaysia for providing the financial support on this project under Project Code GGP-2017-011.

\section{REFERENCES}

[1] Syed Mustaffa S A, Musirin I, Othman M M, Mansor M H. Chaotic Mutation Immune Evolutionary Programming for Voltage Security with the Presence of DGPV. Indonesian Journal of Electrical Engineering and Computer Science, Vol. 6, No. 3, pp. $721 \sim 729,2017$.

[2] Zichun Y et al. Analytical method of the impact of distributed generation on static voltage stability of distribution network and its development. TELKOMNIKA Indonesian Journal of Electrical Engineering, Vol. 11, No. 9, pp. 5018 5029, 2013.

[3] Vovos P N, Kiprakis A E, Wallace A R, Harrison G P. Centralized and distributed voltage control: Impact on distributed generation penetration. IEEE Transaction on Power Systems, 22(1), pp. 476-83, 2007.

[4] Caisheng W, Nehrir MH. Analytical approaches for optimal placement of distributed generation sources in power systems. IEEE Transaction on Power Systems, 19(4), pp. 2068-2076, 2004.

[5] Acharya N, Mahat P, Mithulananthan N. An analytical approach for DG allocation in primary distribution network. International Journal Electric Power, 28(10), pp. 669-78, 2006.

[6] Aman M M, Jasmon G B, Mokhlis H, Bakar A H A. Optimal placement and sizing of DG based on a new power stability index and line losses. Electrical Power and Energy Systems,43, pp. 1296-1304, 2012.

[7] Al Abri R S, El-Saadany Ehab F, Atwa Yasser M. Optimal placement and sizing method to improve the voltage stability margin in a distribution system using distributed generation. IEEE Transaction on Power Systems, 28(1), pp. 326-34, 2013.

[8] Murty V V, Ashwani K. Optimal placement of DG in radial distribution systems based on new voltage stability index under load growth. Electrical Power and Energy, 69, pp. 246-56, 2015.

[9] Kaviani-Arani A. Optimal placement and sizing of distributed generation units using co-evolutionary particle swarm optimization algorithms. TELKOMNIKA Indonesian Journal of Electrical Engineering, Vol. 13, No. 2, pp. 247 256, 2015.

[10] Moravej Z, Akhlaghi A. A novel approach based on cuckoo search for DG allocation in distribution network. Electric Power Energy Systems, 44, pp.672-679, 2013.

[11] Rosseti Gustavo J S, de Oliveira Edimar J, de Oliviera Leonardo W, CSilva I, Peres W. Optimal allocation of distributed generation with reconfiguration in electric distribution systems. Electric Power System Research,103, pp. 178-83, 2013.

[12] Kayal P, Chanda C K. Placement of wind and solar based DGs in distribution system for power loss minimization and voltage stability improvement. Journal on Electrical Power and Energy Systems 53, pp. 795-809, 2013.

[13] Nasiraghdam H, and Jadid S. Optimal hybrid PV/WT/FC sizing and distribution system reconfiguration using multiobjective artificial bee colony algorithm. Journal on Solar Energy, 86, pp. 3057-71, 2012.

[14] Hung D Q, Mithulananthan N, Bansal R C. Integration of PV and BES units in commercial distribution systems considering energy loss and voltage stability. Journal on Applied Energy, 113, pp. 1162-70, 2014.

[15] Bayraktar Z, Komurcu M, Werner D H. Wind driven optimization (WDO): A novel nature-inspired optimization algorithm and its application to electromagnetics 2010.

[16] Yang C F, Lai G G, Lee C H, Su C T, Chang G W. Optimal setting of reactive compensation devices with an improved voltage stability index for voltage stability enhancement. Electrical Power and Energy Systems, 37, pp. 50-57, 2012.

[17] He J, Guo H. A modified particle swarm optimization algorithm. TELKOMNIKA Indonesian Journal of Electrical Engineering, Vol. 11, No. 10, 6209 6215, 2013. 